\title{
On the role of dust in the lunar ionosphere
}

\author{
T. J. Stubbs, ${ }^{1,2,6}$ D. A. Glenar, ${ }^{3,2,6}$ M. R. Collier, ${ }^{2,6}$ W. M. Farrell, ${ }^{2,6}$ \\ J. S. Halekas, ${ }^{4,6}$ G. T. Delory, ${ }^{4,5,6}$ R. R. Vondrak ${ }^{2,6}$ \\ 'Goddard Earth Sciences and Technology Center, University of Maryland, \\ Baltimore County, Baltimore, MD 21228 \\ ${ }^{2}$ NASA Goddard Space Flight Center, Greenbelt, MD 20771 \\ ${ }^{3}$ New Mexico State University, Las Cruces, NM 88003 \\ * Space Sciences Laboratory, University of California, Berkeley, CA 94720 \\ ${ }^{5}$ NASA Ames Research Center, Moffett Field, CA 94035 \\ ${ }^{6}$ NASA Lunar Science Institute, NASA Ames Research Center, Moffett Field, CA 94035 \\ Tmothy, Stubbs@NASA.gov
}

\begin{abstract}
Evidence suggests that electron concentrations above the dayside lunar surface can be significantly higher than expected from either the photo-ionization of exospheric neutrals or any other well-known process. The Luna 19 mission performed dualfrequency radio occultation experiments in order to determine electron column concentrations above the lunar limb as a function of tangent height ${ }^{1}$ (shown in the figure below). The resulting electron concentration profiles surprisingly indicated a peak of $\sim 500-1000 \mathrm{~cm}^{-3}$ and scale heights of $\sim 10-30 \mathrm{~km}$. It has been suggested that electrically charged exospheric dust could contribute to these electron enhancements ${ }^{2}$. Here we describe how to estimate the electrons produced by photo-charged dust, which is then used to predict electron concentrations from exospheric dust distribution models that are based on the "excess brightness" observed in Apollo 15 coronal photographs $^{3}$. The results indicate that radio occultation measurements likely provide a valuable perspective on the role of dust in the lunar environment.

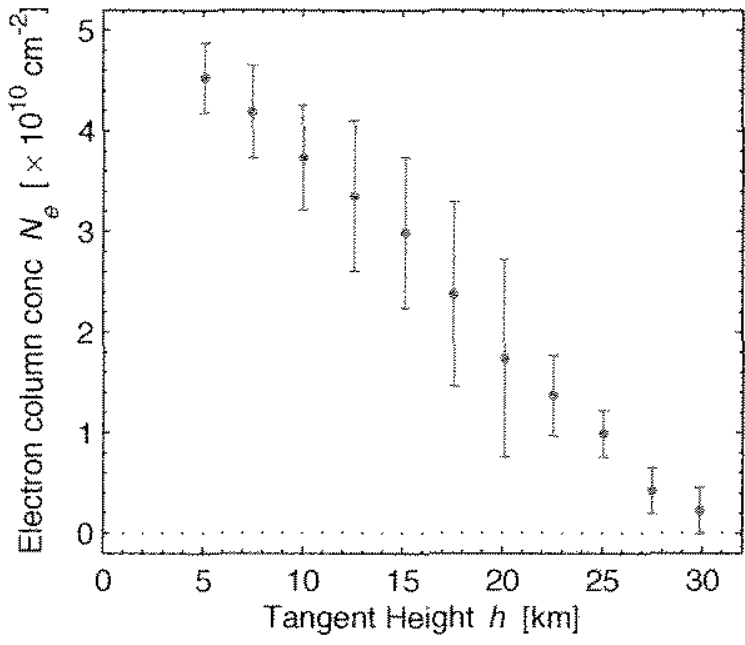

Electron column concentrations measured above the lunar limb by Luna $19^{\prime}$.

\footnotetext{
'Vasil'ev, M. B., et al, Radio transparency of circumlunar space using the Lunam 19 station, Cosmic Research, 12, 102-107 (1974).

${ }^{2}$ Imamura, T, et al., The possibility of studying the lunar ionosphere with the SELENE radio science experiment, Earth Planets Space, 60, 387-390 (2008).

${ }^{3}$ McCoy, J.E., Photometric studies of light scattering above the lunar terminator from Apollo solar corona photography. Proc.7th Lunar Sci. Conf, $1087-1112$ (1976).
} 